\title{
¿Certificación en buenas prácticas éticas de la investigación? Una necesidad para incentivar la producción científica
}

\section{Certification on Good Ethical Practices in Research? A Necessity for Greater Scientific Productivity}

\section{Guillermo Droppelmann 1,2, 3, Felipe Feijoo 4, David Prieto 5,6}

1. Área de Investigación, Unidad Académica, Clínica MEDS, Santiago, Chile.

2. Facultad de Ciencias, Universidad Mayor, Santiago, Chile.

3. Programa de Doctorado en Ciencias mención Estadística Avanzada, Universidad Católica San Antonio, Murcia, España.

4. Escuela de Ingeniería Industrial, Pontificia Universidad Católica de Valparaíso, Valparaíso, Chile.

5. Facultad de Ciencias de la Salud, Universidad Católica San Antonio de Murcia, Murcia, España.

6. Facultad de Epidemiología y Salud Poblacional, London School of Hygiene and Tropical Medicine, Londres, Inglaterra.

\section{Resumen}

La investigación constituye una fuente fundamental para el crecimiento de los países y para el desarrollo de las disciplinas. Diversos son los factores que influyen en la baja productividad científica de profesionales pertenecientes al área de la salud en Chile. Se destacan una serie de elementos obstaculizadores. La creación de un programa de certificación en buenas prácticas éticas de la investigación clínica en estudios cuya naturaleza sea descriptiva, podría fomentar un acercamiento entre los clínicos y los comités de ética científicos, así como el incentivo a una mayor productividad científica.

Palabras clave: Buenas prácticas, Certificación, Ética, Investigación.

\section{Abstract}

Research is a fundamental source for the growth of countries and for the development of disciplines. Several factors influence the low scientific productivity of professionals belonging to the health area in Chile, highlighting a series of obstacles. The creation of a certification program on good ethical practices of clinical research in studies whose nature is descriptive could facilitate the connections between clinicians and scientific ethics committees, as well as being an incentive for greater scientific productivity.

Keywords: Good practices, Certification, Ethics, Research. 
"He leído varios libros sobre los aspectos formales de la ciencia, sobre la filosofía de la ciencia y sobre el método científico, y todos ellos son excelentes para hacer pensar, pero de ningún modo son eficaces para enseñar a descubrir o para crear investigadores".

Santiago Ramón y Cajal. Reglas y consejos sobre la investigación científica. Los tónicos de la voluntad (1897).

\section{Introducción}

La investigación y el desarrollo (I+D) han sido reconocidos por las principales instituciones gubernamentales a nivel mundial como uno de los pilares fundamentales para alcanzar una mejor productividad, crecimiento económico e impacto en el bienestar de las personas, incentivando la colaboración entre países con el propósito de promover las políticas públicas referentes a ciencia y tecnología (6).

La innovación y los avances en el campo de la salud son necesarios para hacer frente a los numerosos problemas multidimensionales e interrelacionados existentes, permaneciendo muchos por largos periodos sin resolver. Para hacer frente a esta problemática, el sistema de atención de salud requiere una masa crítica de individuos capacitados para poder llevar a cabo investigación de calidad, permitiendo impactar con relevancia dentro de la comunidad en la cual se desempeñan (Stevens, 2011).

Uno de los aspectos fundamentales para poder realizar cualquier tipo de investigación biomédica en seres humanos, según la normativa nacional e internacional vigente, es contar con la aprobación de un comité de ética científico (CEC) (Schroter, 2006), debidamente acreditado por la instancia superior de salud para su funcionamiento. Entre las funciones que han de cumplir sus integrantes está la de revisar exhaustivamente una serie de documentos y formularios, con el propósito de emitir un pronunciamiento, permitiendo su autorización o rechazando su posterior ejecución (Garrard, 2005).

Entre los documentos que se solicitan para evaluar la factibilidad técnica y práctica, se encuentra el protocolo de investigación, el cual es un escrito detallado que describe desde los principales argumentos existentes del estado del arte del problema en cuestión, hasta consideraciones metodológicas, alcances éticos y disposiciones prácticas para su completa ejecución.

La magnitud de la extensión de este registro, la solicitud de distintas autorizaciones, los tiempos de resolución y espera (Bueno 2009), la compleja institucionalidad burocrática (Bozeman, 2009), pudieran explicar la falta de interés por parte de la comunidad clínica en investigar, respaldada por las escasas publicaciones de los profesionales que se dedican a la atención y cuidado de pacientes, o incluso sus pobres hallazgos y escasa utilidad clínica (loannidis, 2016).

Es importante hacer una distinción entre las investigaciones de carácter directo, es decir, aquellas que realizan una intervención sobre las personas, e indirecto, cuando las variables de interés son simplemente observadas, pero no son manipuladas sobre los seres humanos. En esta perspectiva, cabe destacar aquellos diseños de investigación de tipo observacional, abordajes temporales retrospectivos 0 
prospectivos, revisión de fichas de pacientes, estudios y series de casos clínicos, que, pese a que son considerados erróneamente con escasa jerarquía desde el punto de vista de la evidencia científica, constituyen la mayoría de las bases estructurales para todos aquellos estudios de mayor complejidad metodológica.

El presente artículo tiene como objetivo plantear la necesidad de contar con una certificación en buenas prácticas éticas de la investigación que permita acelerar y estimular el proceso de investigación por parte de los profesionales pertenecientes a las áreas médicas, biomédicas y ciencias clínicas.

\section{Estado de la investigación en salud en Chile}

\subsection{Investigador clínico}

La Organización para la Cooperación y el Desarrollo Económico (OECD) define como investigadores a aquellos profesionales comprometidos con la concepción y creación de nuevos conocimientos, productos, procesos, métodos, en el interior de sistemas que están directamente involucrados en la gestión de proyectos con igual objetivo pertenecientes a instituciones gubernamentales, académicas, y centros vinculados con la investigación en el sector privado (OECD, 2018). El Instituto Canadiense en Investigación en Salud propone el termino de científico-clínico para designar al individuo que posee un grado profesional en el área de la salud pero que pasa gran parte de su tiempo realizando labores de investigación, sin dejar de lado su responsabilidad asistencial (ICRH, 2002).

En Chile existe un escenario poco propicio para la investigación que involucra la escasez de investigadores clínicos, un marco gubernamental rígido, la falta de formación y de medios para la divulgación.
Desgraciadamente, la mayor parte de los trabajos de investigación en salud nunca se incorporan en la práctica clínica (Estabrooks, 2006; Scott, 2008). Del mismo modo, muchos de los problemas clínicos raramente se traducen en proyectos concretos de investigación (Jansson, 2010). Esta brecha se debe, en gran parte, a una falta de participación de los médicos y profesionales del área de la salud en investigación científica competitiva, lo que dificulta su evaluación crítica de los artículos que lee y le priva de conocimiento que podría obtener eventualmente de los nuevos avances de sus disciplinas, pudiendo incluso prescribir tratamientos que en la actualidad han sido reportados como dañinos (Jiwa, 2012). Los profesionales sanitarios que logran ser también investigadores según estándares internacionales, representan un recurso humano absolutamente necesario para las escuelas de ciencias de la salud, pero se convierten en un recurso costoso de mantener o renovar (Salas, 2005), debido a que los programas académicos existentes destinados a la formación de capital humano avanzado, no han fomentado el desarrollo de estas importantes competencias investigadoras (IDRC/CONICYT).

Para solucionar esta brecha existente entre la investigación en salud y la práctica clínica, debiera promoverse una mayor cantidad de profesionales que desarrollen permanentemente nuevos conocimientos relevantes para la atención clínica. Este profesional/científico-investigador debe ser un sujeto comprometido con la búsqueda de nuevos conocimientos, herramientas y enfoques que le permitan obtener el mejor diagnóstico, tratamiento y prevención de las diferentes enfermedades existentes 
(Lawrence, 2010), logrando llenar el vacío entre la práctica profesional y la investigación, traduciendo los resultados de la investigación en la clínica y definiendo los asuntos de investigación basados en temas clínicos que se enfrentan en las labores cotidianas (Ben-David, 1960; Roy, 1997).

\subsection{Marco gubernamental}

El marco regulatorio legislativo existente en Chile tampoco favorece la producción de investigación en el área de la salud, lo cual ha sido motivo de constante cuestionamiento por parte de la comunidad científica y académica del país (Oyarzún, 2013; Vega, 2015).

Entre las principales leyes que se destacan en esta temática se encuentra la Ley 20.120 sobre la Investigación Científica en el Ser Humano, su Genoma, que prohíbe la clonación humana, la Ley 19.628 sobre Protección de la Vida Privada, la Ley 20.584 sobre Derechos y Deberes que tienen las Personas en Relación con Acciones Vinculadas a su Atención en Salud y la Ley 20.850 sobre la Protección Financiera para Diagnósticos y Tratamientos de Alto Costo (Ley 19.628), (Ley 20.120) (Ley 20.584), (Ley 20.850), las cuales restringen de alguna forma el incentivo de áreas de investigación que resultan fundamentales para el avance del conocimiento y el mejoramiento de ciertas poblaciones más vulnerables.

A nivel gubernamental, Chile no contaba hasta el año pasado con un Ministerio de Ciencia e Investigación, ni con una política nacional que fomentara la investigación intersectorial,

Son varios los elementos obstaculizadores que desincentivan la realización de investigación por parte del profesional clínico. aunque sí posee diversas instituciones que se encargan de la administración y distribución de los recursos financieros destinados por el Estado para el incentivo de la investigación en salud. Destaca la Comisión Nacional de Investigación Científica y Tecnológica (CONICYT) (CONICYT, 2018) del Ministerio de Educación, que a través del Fondo de Desarrollo de Ciencia y Tecnología (FONDECYT), institución estatal dedicada a estimular y promover el desarrollo de la investigación científica y tecnológica del país (FONDECYT, 2018), y del Fondo de Fomento al Desarrollo Científico y Tecnológico (FONDEF) (FONDEF, 2018), que en conjunto con el Ministerio de Salud conforman el Fondo Nacional de Investigación en Salud (FONIS) (FONIS, 2018), que tienen como propósito la promoción de la investigación aplicada en salud de alta calidad, alineándose con el cumplimiento de los objetivos estratégicos de la década en salud (MINSAL, 2018). No obstante, la forma de postulación a estos fondos resulta muchas veces engorrosa para aquellos profesionales clínicos no habituados a este tipo de actividades.

El esfuerzo gubernamental parece no ser suficiente, ya que según el informe del CONICYT realizado durante el año 2016, las áreas de mayor producción científica durante el periodo comprendido entre 2000-2015 fueron la astronomía, la biología molecular, la ecología, la física de partículas y la biología marina (CONICYT, 2018), no existiendo registro en el área de las ciencias médicas, de la salud o clínicas.

\subsection{Formación de capital humano}

A nivel de educación superior, el escenario destaca por una contribución creciente de diversos centros de investigación y de estudios avanzados, destacando las 
universidades estatales adheridas al Consejo de rectores de las universidades chilenas $(\mathrm{CRUCH})$, que presentan los mejores indicadores, lo que a su vez explica que la gran mayoría de las investigaciones se caractericen por su fuerte carácter académico más que por el abordaje clínico. Eso a su vez pone en evidencia los grandes problemas existentes, tanto con las fuentes de financiamiento como con la formación de los equipos humanos de investigación (Solimano, 2011).

Dentro de la formación de profesionales, destacan algunas iniciativas académicas, como los programas de doctorado en ciencias médicas de la Universidad de Chile, de la Pontificia Universidad Católica de Chile y de la Universidad Austral, así como las más recientes de la Universidad de la Frontera y la Universidad Del Desarrollo. Sin embargo, aún no existe una oferta académica importante por parte de las instituciones de educación superior y de las mismas instituciones públicas y privadas de salud, en orden a satisfacer esta imperiosa necesidad de desarrollar la investigación en el área clínica, ya que los esfuerzos se concentran principalmente en la formación de sujetos que puedan dar respuesta a la creciente demanda asistencial, educando a especialistas y subespecialistas con escaso entrenamiento en investigación. Los registros demuestran el escaso interés de los profesionales de la salud en acceder a programas académicos de doctorado (CONICYT, 2000).

Esta situación ha permanecido prácticamente invariable con el transcurso del tiempo, ya que durante la última convocatoria de becas de doctorado otorgadas por CONICYT a nivel nacional, las ciencias médicas y de la salud se encontraban nuevamente en el último lugar con un 5\% de los beneficiarios (CONICYT, 2018).

\subsection{Divulgación científica}

Si se considera como indicador la cantidad de literatura científica publicada en el área de la salud, existe un mejor posicionamiento, sobresaliendo incluso por encima de las ciencias naturales, ingeniería y tecnología, las ciencias agrarias, así como las ciencias sociales y humanidades, pero si se observa la cantidad de citaciones de los documentos, el número es más bajo que algunas de las disciplinas mencionadas, y si se compara con un indicador internacional estandarizado como el impacto de la citación, el número desciende aún más (CONICYT, 2018).

Otro indicador que podría resultar de interés es la ubicación de las revistas chilenas en el Journal Citation Reports (JCR). En él solo existían revistas chilenas del área de las ciencias médicas y de la salud en el cuartil 4, ascendiendo al cuartil 3 si al ubicarlas en el SCImago Journal Rank de igual año (CONICYT, 2018). Esto evidencia no solo un déficit en la cantidad de las publicaciones en esta área, sino que también en su calidad.

Según FONDECYT, la cantidad de proyectos que se adjudicaron a través de fondos de financiamiento público para el área de medicina y ciencias biomédicas, no supera en promedio 24 proyectos por año en el periodo regular 2014 y 2018, quedando posicionada por debajo de otras áreas (FONDECYT, 2018). Son manifiestas las desigualdades entre instituciones públicas y privadas de educación superior, así como entre zonas geográficas, repitiéndose la situación ya registrada en el periodo comprendido entre los años 1980 - 2000 (CONICYT, 1981-2000). De nuevo resulta palpable la escasez de proyectos dedicados específicamente a mejorar la calidad de la salud de las personas. 


\section{BUROCRATIZACIÓN DE LA INVESTIGACIÓN}

\subsection{Contexto histórico}

La investigación, la creación y el aprendizaje se limitan cuando se reglamentan excesivamente los procesos mediante extensos protocolos y se rigidizan las operaciones naturales que las incentivan, provocando un detrimento de su desarrollo (Tejeda, 2013).

El término burocracia presenta diversas connotaciones conceptuales. Nosotros la definimos como un sistema de organización ineficiente con trámites lentos y costos, sin flexibilidad en sus normas de actuación (Vargas, 2009). Esto implica un gran esfuerzo a los investigadores, exigiéndoles la cumplimentación de formularios que carecen de utilidad y sometiéndoles a un proceso fiscalizador agobiante, que muchas veces posiciona a los científicos bajo sospecha innecesaria (González, 2004).

Este escenario en ningún caso es azaroso, ya que hunde sus raíces en la historia, ya que a lo largo de ella se han presentado innumerables hechos atentatorios contra la dignidad e integridad de los derechos humanos, sobre todo por la falta de ética entre los investigadores científicos de finales del siglo XIX y primer tercio del siglo XX, más incentivados por probar sus hipótesis que por velar por la integridad de las personas (Sierra, 2011).

Tal fue el caso de los experimentos criminales realizados por Giuseppe Sanarelli tras inocular con fiebre amarilla a personas (Sanarelli, 1897), las intervenciones hechas por Carlos Finlay y la Comisión Walter Reed para determinar la fuente de contagio de la fiebre amarilla (Finlay, 1965; Reed, 1900), la infección deliberada con la bacteria del cólera a prisioneros en Filipinas (Chernin, 1989), las investigaciones de Joseph Goldberg para conocer la evolución natural de la pelagra y determinar un potencial tratamiento (Etheridge, 1993), las múltiples atrocidades ejecutadas por el tercer Reich durante la segunda guerra mundial para conocer los límites del cuerpo humano (Baumslag, 2005; Berenbaum, 1993), los estudios con hepatitis en la escuela de Willowbrook State, infectando a niños con retraso mental para identificar la etiología de la heptatitis (Giles, 1969), la inoculación deliberada a personas con sífilis en Guatemala (Tanne, 2010), así como el estudio realizado en la comunidad de Tuskegee, Alabama en Estados Unidos, denegando el tratamiento a enfermos de sífilis (Carmack, 2008).

Estas atrocidades motivaron a la comunidad científica internacional a desarrollar diversas normativas éticas que protegieran la integridad de los seres humanos, destacando entre ellas el Código de Nüremberg tras la segunda guerra mundial (Nuremberg Code, 1947), el pronunciamiento de la Iglesia Católica a través del papa Pío XIl sobre la investigación en seres humanos (Pío XII, 1952), la declaración de Helsinki propuesta por la Asociación Médica Mundial (AMM) el año 1964 y sus múltiples actualizaciones realizadas en los años 1975, 1983, 1989, 1996, 2000, 2002, 2004, 2008, 2013 (WMA, 1997), el Informe Belmont del año 1979 que describe los principios y guías éticas para la protección de los sujetos humanos de investigación (Gobierno mexicano, 2018), así como las guías elaboradas por el Consejo de Organizaciones Internacionales de las Ciencias Médicas (CIOMS) (CIOMS, 2018), y los esfuerzos individuales de algunos médicos bioeticistas tales como Ezequiel Enmanuel (Emanuel, 2008). 
Cabe destacar que muchos investigadores no siguen estas recomendaciones (Suárez, 2015), ya sea por desconocimiento o simplemente porque constituye un obstáculo más en el proceso de investigación. La situación cambia y se ve influenciada en mayor medida gracias a las exigencias impuestas recientemente por los comités editoriales de las revistas científicas internacionales, al exigir la aprobación de un ente regulatorio, comité, que no solo debe actuar como un evaluador externo al equipo de investigadores (ICMJE, 1997; Amdur, 1997), sino que también ha de verificar la factibilidad metodológica de los protocolos de investigación que se presentan (Droppelmann, 2018), así como valorar el verdadero impacto y utilidad para las personas que serán sometidas a una determinada intervención. De esta forma se establece la obligatoriedad de contar con algún tipo de pronunciamiento ético, incrementando nuevamente la complejidad en la ejecución de un proyecto de investigación (UNESCO, 2006).

En Chile, la creación de los comités de ética científicos (CEC), tanto en servicios de salud regionales como en hospitales públicos y privados, surgen a través de la creación de la Norma 57 del Ministerio de Salud en el año 2001, que regula el correcto procedimiento para la implementación de los ensayos clínicos de productos farmacéuticos en seres humanos (ISP, 2018).

\subsection{Aspectos obstaculizadores}

Dentro de los aspectos institucionales que hemos identificado como obstaculizadores del proceso de investigación biomédica, aparte de la ya mencionada burocracia administrativa, se encuentra la extensa documentación solicitada por los CEC (tabla1), que incluye la recomendada por la Organización Panamericana de la Salud (OPS, 2011).

Si bien esa documentación resulta siempre necesaria, debería adecuarse en detalle y extensión a las características propias de cada tipo de diseño. Por ejemplo, la documentación requerida para un proyecto con diseño observacional retrospectivo debería ser menos extensa y detallada que la exigida para aprobar un estudio cuyo propósito sea intervenir a un grupo de personas para probar una sustancia con fines terapéuticos a través de un ensayo clínico.

Tabla 1. Documentos que solicita un comité de ética científico.

1. El formulario de solicitud fechado y firmado por todos los solicitantes enumerados y funcionarios institucionales.

2. El protocolo del proyecto de investigación propuesto, identificado y fechado claramente, junto con la documentación y los anexos.

3. Un resumen o sinopsis del proyecto en un lenguaje no técnico.

4. Una descripción (que se puede incluir en el protocolo) de las consideraciones éticas que implica la investigación propuesta.

5. Información sobre investigaciones anteriores en la misma área de trabajo que justifique o apoye la propuesta.

6. Un resumen adecuado de todos los datos de seguridad, farmacológicos, farmacéuticos y toxicológicos disponibles sobre el producto sujeto a estudio, junto con un resumen de la experiencia clínica con ese producto hasta la fecha, cuando la investigación incluya 
un producto experimental.

7. Los curricula vítae actualizados de los principales investigadores.

8. Todos los formularios de recopilación de datos que se utilizarán en el proyecto de investigación.

9. Todos las formularios, documentos y anuncios que se utilizarán para la captación de potenciales participantes.

10. Una descripción detallada del proceso y las estrategias de captación.

11. Los formularios de consentimiento informado.

12. Una descripción del proceso que se usará para obtener y verificar el consentimiento informado.

13. Una descripción de las medidas que se adoptarán para garantizar la protección de la privacidad de los participantes y la confidencialidad de los datos.

14. Una declaración de las remuneraciones u otros productos o servicios que se proporcionarán a los participantes en el estudio.

15. Una descripción de los acuerdos de cobertura de seguro para los participantes en la investigación, cuando corresponda.

16. Todas las decisiones anteriores de otros CEC $\circ$ autoridades reguladoras sobre el estudio propuesto.

17. Una declaración de que los investigadores están totalmente de acuerdo en cumplir los principios éticos planteados en las pautas pertinentes.

Otro punto importante que podría influir en el desinterés de los clínicos por la investigación, es el tiempo que transcurre desde la presentación del proyecto hasta la obtención de la aprobación para su ejecución. El tiempo promedio de aprobación es 30 a 60 días (Novaes, 2009), no muy distante a lo que propone la autoridad sanitaria de Chile a través del reglamento de comités de ética científica, que exige un plazo no mayor de 45 días, prorrogable 20 días más por razones previamente justificadas (MINSAL, 2018). Estos son los tiempos para estudios de intervención tipo ensayo clínico. No existen datos sobre los tiempos de respuesta para aquellos estudios observacionales.

\section{CERTIFICACIÓN EN BUENAS PRÁCTICAS ÉTICAS EN INVESTIGACIÓN}

\subsection{Entrenamiento en bioética o ética clínica}

Un aspecto fundamental es la formación por parte de los estudiantes y profesionales del área de ciencias médicas, biomédicas y de la salud en temas relacionados con la ética de la investigación. Pese a que la mayoría de las instituciones clínicas y educacionales declaran que existe una relación compartida entre educar e investigar (Annas, 1980), se necesita la integración de los conocimientos teóricos recibidos (Cadicott, 2005) con la práctica de los profesionales (Gü Ican, 2015), y que a su vez la entrega de conocimientos sea lo más homogénea posible en el conjunto de las instituciones (Ferreira-Padilla, 2016), buscando siempre el mejoramiento de las personas objeto de estudio. 
Es común el acuerdo de que un equilibrio entre la capacitación del personal del área de la salud y el respeto de la autonomía del paciente puede dar como resultado un mejor rendimiento en términos de enseñanza, práctica e investigación (Ramana, 2013). De ahí la importancia moral de la formación y el entrenamiento (Savulescu, 1996). Es por este motivo que en la última década surgió la necesidad de contar con normas en buenas prácticas en investigación clínica. A instancias de organizaciones internacionales tales como la Conferencia Internacional de Armonización la Comunidad Europea o la Organización Mundial de la Salud, y de países como Estados Unidos y Japón, se han desarrollado "Guías de Buenas Prácticas Clínicas" (GBPC). Estas guías definen una serie de pautas y recomendaciones para el diseño, realización, auditoría, análisis e informe de estudios clínicos, de tal modo que se garantice su calidad y fiabilidad (OPS, 2018).

Resulta fundamental alcanzar un equilibrio entre formación de los profesionales de la salud, actividad asistencial y desarrollo de la investigación, a fin de aumentar la calidad de vida y mejorar el estado de salud de las personas.

quiera hacer investigación con seres humanos debe seguir un programa de educación en ética de la investigación. Así surgió en el año 2000 el Programa de Educación en Ética de la Investigación o, por sus siglas originales en inglés, Collaborative Institutional Training Initiative (CITI), el cual ofrece una serie de cursos de formación para profesionales (CITI, 2018).

En Chile aún no se exige en la actualidad formación específica en ética científica al momento de investigar en seres humanos y solo se dispone de normas y regulaciones que permiten complementar las disposiciones vigentes en las leyes 20.120 y 20.584 , según las cuales toda intervención física o psíquica o interacción con seres humanos con el objetivo de mejorar la prevención, diagnóstico, tratamiento, manejo y rehabilitación de la salud de las personas o de incrementar su conocimiento biológico, debe contar con la aprobación de un comité de ética científico (Ley 20.120, Ley 20.584).

Por otro lado, las instancias creadas para la capacitación de los profesionales e investigadores, consideran al ensayo clínico como referente para el desarrollo de la investigación, no abordando ningún tipo de estudios de características observacionales (Parab, 2010). A nuestro entender este tipo de estudios, así como la revisión de información retrospectiva, constituyen herramientas fundamentales de la investigación clínica (Grimes, 2002), por lo que su utilidad no debe ser menospreciada ni relegada a posiciones de escaso nivel de evidencia. No hay duda de que el ensayo clínico es el gold standard. Pero, se hace importante y necesario distinguir entre aquellas investigaciones que poseen un carácter prospectivo y las de tipo retrospectivo (Oyarzún, 2014)

Todo profesional perteneciente al área de la salud, ciencias biomédicas y clínicas que presente interés en desarrollar conocimiento con información proveniente de seres humanos, debiera contar con al menos algún tipo formación académica de postgrado en temas asociados con la ética de la investigación. Creemos que aquellos clínicos 
que poseen interés en investigar en temas relacionados con su práctica cotidiana, deberían contar obligatoriamente con una certificación en buenas prácticas éticas de la investigación, que les permita desarrollar propuestas de investigación sin tener que presentar cada vez la extensa documentación que se exige actualmente. Para mantener al investigador actualizado, podría exigirse que la certificación se renovase cada cierto número de años.

El investigador que tuviera esta acreditación de que cumple con los estándares comunes para investigar, podría presentar al comité de ética una documentación más resumida cuando no existiese intervención directa en seres humanos, haciendo también más expedita la labor del comité.

Poseer una certificación en buenas prácticas éticas de la investigación clínica podría facilitar los procesos e incentivar la investiqación. en investigar. Además, les permitiría presentar de forma más rápida y ejecutiva proyectos de investigación relacionados con su actividad clínica.

La posibilidad de presentar protocolos abreviados, más el aval de que posee las competencias necesarias, permitiría facilitar al clínico el acceso a la investigación en el área de su especialidad, lo que repercutirá no solo en el número y calidad de las publicaciones, sino también en la mejora de su actividad profesional, que a su vez tendrá efectos positivos en las personas y enfermos a los que atiende cotidianamente.

\subsection{Propuesta de certificación en buenas prácticas éticas de la investigación clínica}

Los siguientes aspectos resultan fundamentales para sustentar nuestra propuesta.

El primero es que tanto hospitales como instituciones de salud poseen profesionales bien intencionados, dentro de cuyos objetivos de trabajo se encuentre entregar el mejor cuidado posible a los pacientes (Agich, 1991).

El segundo punto se basa en que los comités de éticas científicos se transformen en parte integral de las estructuras organizacionales de los servicios de salud, universidades e institutos de investigación (Doyal, 2001), resultando cada vez más habitual su presencia al interior de las instituciones.

El tercer aspecto lo constituyen los integrantes de los comités de ética científica, conformados por profesionales con competencias y destrezas únicas, muchas veces vinculadas fuertemente con la academia. El implementar programas de certificación en buenas prácticas éticas de la investigación al interior de los comités para los investigadores interesados, resultaría altamente necesario.

Proponemos que la iniciativa de certificación sea otorgada por los comités de ética científicos acreditados por la autoridad sanitaria respectiva o por aquellos comités que se encuentran en vías de acreditación según la legislación nacional actual. Que éstos asuman un rol no solo fiscalizador de los proyectos de investigación, sino que se transformen en entes que favorezcan la investigación actuando como puentes entre los investigadores y las investigaciones. 
El Departamento de bioética del Instituto Nacional de Salud de Estados Unidos (NIH), propone un entrenamiento en ética y una regulación de los aspectos relacionados con la investigación clínica para cualquier profesional que quiera obtener mayores conocimientos acerca de esta temática. $(\mathrm{NIH}, 2018)$ Creemos que los cinco primeros objetivos son perfectamente aplicables a nuestra propuesta. Mencionamos algunos otros objetivos que resultan fundamentales para poder obtener un entrenamiento sólido en ética de la investigación y una posterior certificación en buenas prácticas éticas de la investigación. (ver Tabla 2).

Tabla 2. Objetivos comunes de aprendizaje para un programa de entrenamiento en bioética para clínicos.

1. Conocer el marco sistemático para evaluar la ética de un protocolo de investigación clínica.

2. Aplicar códigos apropiados, regulaciones y otros documentos que rigen la conducta ética de los investigadores y de los sujetos que se someten a investigación.

3. Discutir temas controvertidos relacionados con la investigación en seres humanos.

4. Identificar aspectos críticos del consentimiento informado y desarrollar estrategias para su correcta implementación.

5. Analizar la experiencia de sujetos que han participado en protocolos de investigación.

6. Identificar aspectos básicos en el manejo de fichas clínicas y bases de datos.

7. Desarrollar estrategias de resguardo de información confidencial de personas y pacientes.

8. Valorar la investigación como un proceso de mejoramiento de la salud de las personas de los enfermos y de la gestión institucional.

9. Acercar la investigación a los profesionales del área de la salud.

10. Reconocer al comité de ética científico como un facilitador entre la investigación y los investigadores.

\section{REFLEXIONES FINALES}

La investigación en salud es esencial para el progreso de los países (WHO, 2018) y el ámbito clínico resulta fundamental para buscar la mejor evidencia de los procedimientos utilizados y complementar la experiencia clínica de los profesionales (Gálvez, 2003).

La investigación científica en seres humanos siempre ha planteado el conflicto entre la práctica clínica y la generación de conocimiento producto de esta misma actividad (Novoa, 2007). A pesar de lo cual, la investigación resulta imprescindible para mejorar el proceso diagnóstico-terapéutico del paciente y mejorar la salud de las personas, incrementando de forma continua y paulatina la creatividad por parte de los profesionales que desarrollan actividades asistenciales (Otzen, 2017) y estimulando en los profesionales el pensamiento crítico, la actitud proactiva, innovadora y fomentando la curiosidad personal y laboral (Ruiz, 2010).

Entre las limitaciones que puede encontrarse un profesional al momento de investigar, se encuentran no solo las relacionadas con los aspectos metodológicos (Henríquez, 
2003), institucionales, gubernamentales y de formación, sino también aquellas propias de la ética (Rodríguez, 2002).

Creemos necesario llevar a cabo futuros estudios que permitan conocer las principales impresiones de los distintos participantes en torno a la factibilidad técnica y operativa de implementar un programa que certifique las buenas prácticas éticas en investigación en el área de la salud en Chile. De esta forma se podrán lograr dos propósitos, el primero acercar a los clínicos e investigadores a la "cuestión ética" y lo segundo poder acelerar los procesos para fomentar la investigación al interior de los equipos clínicos.

\section{Bibliografía}

Agich G J, Youngner S J (1991). For experts only? Access to hospital ethics committees. Hastings Cent Rep; 2117-25.

Amdur RJ, Biddle C (1997). Institutional Review Board approval and publication of human research results. JAMA; 277: 909-914.

Annas G J (1991). Ethics committees: from ethical comfort to ethical cover. Hastings Cent Rep; 2118-21.

Annas GJ (1980). The care of private patients in teaching hospitals: legal implications. Bull NY Acad Med; 56, 403-11.

Baumslag N (2005). Murderous Medicine: Nazi Doctors, Human Experimentation, and Typhus. Praeger Publishers.

Ben-David J (1960). Roles and innovations in medicine. The American Journal of Sociology; 65, 557-68.

Berenbaum M (1993). The world must know: the history of the Holocaust as told in the United States Holocaust Memorial Museum. Boston: Little Brown; 194-5.

Bozeman B, Slade C, Hirsch P (2009). Understanding Bureaucracy in Health Science Ethics: Toward a Better Institutional Review Board. American Journal of Public Health; 99(9), 1549-1556.

Bueno M, Brevidelli M-M, Cocarelli T, et al. (2009). Reasons for resubmission of research projects to the research ethics committee of a University Hospital in São Paulo, Brazil. Clinics; 64(9), 831-836.

Cadicott CV, Faber-Langendoen K (2005). Deception, discrimination, and fear of reprisal: lessons in ethics from third-year medical students. Acad Med; 80(9), 866-873.

Carmack HJ, Bates BR, Harter IM (2008). Narrative constructions of health care issues and policies: The case of President Clinton's apology-by-proxy for the Tuskegee syphilis experiment. J Med Humanit; 29, 89-109.

Chernin E (1989). Richard Pearson Strong and the iatrogenic plague disaster in Bilibid Prison, Manila, 1906. Rev Infect Dis; 11(6), 996-1004.

CIOMS. Council for International Organizations of Medical Sciences. [consultado 4 Junio 2018]. Disponible en: https://cioms.ch/. 
CITI Program. Research Ethics and Compliance Training. [consultado 10 Junio 2018]. Disponible en: https://about.citiprogram.org/es/homepage-final/

Comisión Nacional de Investigación Científica y Tecnológica (CONICYT). Disponible en: http://www.conicyt.cl/. Consultado el 4 de mayo del 2018.

Comisión Nacional de Investigación Científica y Tecnológica (CONICYT). Áreas más desarrolladas por investigaciones chilenas. Disponible en: http://www.conicyt.cl/blog/2016/04/14/areas-mas-desarrollada-por-investigacioneschilenas-en-web-of-sciences/. Consultado el 1 de mayo del 2018.

Comisión Nacional de Investigación Científica y Tecnológica (CONICYT). Panorama Científico. Edición especial. Mayo 2000. Concurso nacional de proyectos FONDECYT 2000.

Comisión Nacional de Investigación Científica y Tecnológica (CONICYT). Becas para Estudios de Postgrado en Chile. Disponible en: http://www.conicyt.cl/becasconicyt/2017/02/16/735-becas-son-adjudicadas-por-conicyten-su-concurso-de-doctorado-nacional-2017/. Consultado el 3 de mayo del 2018.

Comisión Nacional de Investigación Científica y Tecnológica (CONICYT). Productividad de Universidades $\mathrm{CRUCH}$ según Áreas OCDE. Disponible en: http://www.conicyt.cl/blog/2016/02/05/productividad-de-universidades-cruch-segunareas-ocde/. Consultado el 1 de mayo del 2018.

Comisión Nacional de Investigación Científica y Tecnológica (CONICYT). Ranking Internacional de Revistas Chilenas. Disponible en: http://www.conicyt.cl/blog/2016/01/21/ranking-internacional-de-revistas-chilenas/. Consultado el 1 de mayo del 2018.

CONICYT. Programa FONDECYT: Impacto y desarrollo 1981-2000.

Doyal $L$ (2001). Clinical ethics committees and the formulation of health care policy. $J$ Med Ethics; 2744-49.

Droppelmann G (2018). La instrumentalización Metodológica en la Ética de la Investigación. EIDON 49, 102-114.

Emanuel E (2008). “An Ethical Framework for Biomedical Research@. In: Emanuel E, Grady C, Crouch R, Lie R, Miller F, Wendler D, editors. The Oxford Textbook of Clinical Research Ethics. New York: Oxford University Press; 123-35.

Estabrooks CA, Thompson DS, Lovely JJE, Hofmeyer A (2006). A guide to knowledge translation theory. Journal of Continuing Education in the Health Professions; 26, 2536.

Etheridge E (1993). "Pellagra". En: Kiple KF, editor. The Cambridge World History of Human Diseases. Cambridge: Cambridge University Press; 918-23.

Fernando Suárez Obando (2015). Un marco ético amplio para la investigación científica en seres humanos: más allá de los códigos y las declaraciones. La propuesta de Ezekiel J. Emanuel. Pers.bioét, 19(2), 182-197.

Ferreira-Padilla G, Ferrández-Antón T, Lolas-Stepke F, Almeida-Cabrera R, Brunet J, Bosch-Barrera $J$ (2016). Ethics competences in the undergraduate medical education curriculum: the Spanish experience. Croatian Medical Journal; 57(5), 493-503. 
Finlay C (1965). "El mosquito hipotéticamente considerado como agente de transmisión de la fiebre amarilla". En: Finlay CJ. Obras completas. La Habana: Academia de Ciencias de Cuba / Museo Histórico de las Ciencias Médicas "Carlos J. Finlay"; 247-61.

Fondo de Desarrollo de Ciencia y Tecnología (FONDECYT). Disponible en: http://www.conicyt.cl/fondecyt/. Consultado el 4 de mayo del 2018.

Fondo de Fomento al Desarrollo Científico y Tecnológico (FONDEF). Disponible en: http://www.conicyt.cl/fondef/. Consultado el 4 de mayo del 2018.

Fondo Nacional de Desarrollo Científico y Tecnológico (FONDECYT). Concurso FONDECYT Regular. Disponible en: http://www.conicyt.cl/fondecyt/sobrefondecyt/que-es-fondecyt/. Consultado el 2 de mayo del 2018.

Fondo Nacional de Investigación en Salud (FONIS). Disponible en: http://www.conicyt.cl/fonis/. Consultado el 4 de mayo del 2018.

Gálvez, A. 2003 Un enfoque crítico para la construcción de una enfermería basada en la evidencia. Investigación y Educación en Enfermería. Universidad de Antioquia; $21(1), 50-64$.

Garrard E, Dawson A (2005). What is the role of the research ethics committee? Paternalism, inducements, and harm in research ethics. Journal of Medical Ethics; $31(7), 419-423$.

Giles JP, McCollum RW, Berndtson LW, Krugman S (1969). Viral Hepatitis - Relation of Australia/SH Antigen to the Willowbrook MS-2 Strain. N Engl J Med; 281, 119-22.

Gobierno de Chile. Instituto de Salud Pública. Norma Técnica N57. Regulación de la Ejecución de Ensayos Clínica que Utilizan Productos Farmacéuticos en Seres Humanos. [consultado 9 Junio 2018]. Disponible en: http://www.conicyt.cl/fonis/files/2013/03/Norma-T\%C3\%A9cnica-57.pdf

Gobierno de Chile. Ministerio de Salud. Estrategia Nacional de Salud para el Cumplimiento de los Objetivos Sanitarios de la Década 2011-2020. Disponible en: http://www.salud-e.cl/biblioteca/estrategia-sanitaria-chilena/objetivos-sanitarios-20112020/. Consultado el 4 de mayo del 2018.

Gobierno Mexicano. Informe Belmont. [consultado 4 Junio 2018]. Disponible en: http://www.conbioetica-

mexico.salud.gob.mx/descargas/pdf/normatividad/normatinternacional/10. INTL Infor me Belmont.pdf

González C (2004). La Investigación Básica. La Investigación en Ciencias Fisiológicas: Bioquímica, Biología Molecular y Fisiología. Cuestiones Previas. Educ. méd; 7, 41-50.

Grimes Da (2002). Descriptive studies: what they can and cannot do. Lancet; 359(9301), 145-9.

Henríquez E, Zepeda MI (2003). Preparación de un proyecto de investigación. Ciencia y enfermería; 9(2): 23-28.

IDRC/CONICYT. Science, technology and innovation programs and policies in Chile. Informe final de la misión internacional. 1998-1999. 
Institute of Circulatory and Respiratory Health (2002). The Clinician-Scientist: Yesterday, Today and Tomorrow. Canadian Institute of Health Research.

International Committee of Medical Journal Editors (1997). Uniform Requeriments for Manuscripts Submitted to Biomedical Journals. JAM; 277, 927-934.

Ioannidis JPA (2016). Why Most Clinical Research Is Not Useful. PLoS Medicine; 13(6), e1002049.

Jansson SM, Benoit C, Casey L, Phillips R, Burns D (2010). In for the long haul: Knowledge translation between academic and non-profit organisations. Qualitative Health Research; 10, 131-43.

Jiwa M (2012). Doctors and Medical Science. The Australasian Medical Journal; 5(8), 462-467.

Lawrence F. Brass (2010). Are MD-PhD Programs Meeting Their Goals? An Analysis of Career Choices Made by Graduates of 24 MD-PhD Programs. Academic Medicine; 85(4).

Ley 19.628. Sobre protección de la vida privada [consultado 2 Mayo 2018]. Disponible en: https://www.leychile.cl/Navegar?idNorma=141599.

Ley 20.120. Sobre la investigación científica en el ser humano, su genoma, y prohíbe la clonación humana [consultado 2 Mayo 2018]. Disponible en: https://www.leychile.cl/Navegar?idNorma=253478\&idVersion=2006-09-22.

Ley 20.584. Regula los derechos y deberes que tienen las personas en relación con acciones vinculadas a su atención en salud [consultado 2 Mayo 2018]. Disponible en: http://www.leychile.cl/Navegar?idNorma=1039348.

Ley 20.850. Crea un sistema de protección financiera para diagnósticos y tratamientos de alto costo [consultado 2 Mayo 2018]. Disponible en: https://www.leychile.cl/Navegar?idNorma=1078148\&buscar=Ley+ricarte+soto.

Ministerio de Salud. Ley 20.120. Sobre la investigación científica en el ser humano, su genoma, y prohíbe la clonación humana. 2006. [consultado 10 Junio 2018]. Disponible en: https://www.leychile.cl/Navegar?idNorma=253478.

Ministerio de Salud. Ley 20.584. Derechos y Deberes de las Personas en Atención de Salud; Acciones Vinculadas a la Atención en Salud. 2012. [consultado 10 Junio 2018]. Disponible en: https://www.leychile.cl/Navegar?idNorma=1039348.

MINSAL. Reglamento Comités de Ética Científica. consultado 9 Junio 2018]. Disponible http://www.minsal.cl/portal/url/item/50e6cfdfbb26b87be04001011e012efe.doc.

$\mathrm{NIH}$. The department of bioethics. [consultado 5 Julio 2018]. Disponible en: https://www.bioethics.nih.gov/courses/ethical-regulatory-aspects.shtml.

Novaes MRG, Guilhem D, Lolas F (2009). Ethical conduct in research involving human beings in Brazil: Diagnosis of research ethics committee. Arq Med; 23(4), 145-50.

Novoa S Fernando (2007). Desafíos éticos en la investigación y aplicación clínica de la genética. Rev. chil. neuro-psiquiatr; 45(4), 305-313. 
Nur Yeliz Gülcan (2015). Discussing the Importance of Teaching Ethics in Education. Procedia - Social and Behavioral Sciences; 174, 2622-2625.

OECD. OECD Global Science Forum. [consultado 4 Junio 2018]. Disponible en: http://www.oecd.org/sti/sci-tech/oecdglobalscienceforum.htm.

OECD. Sciencie and Tecnology. [consultado 4 Junio 2018]. Disponible en: http://www.oecd.org/sdd/08 Science and technology.pdf.

Organización Panamericana de la Salud. Buenas Prácticas Clínicas: Documento de las Américas. [consultado 10 Junio 2018]. Disponible en: http://apps.who.int/medicinedocs/documents/s18627es/s18627es.pdf.

Organización Panamericana de la Salud (2011). Pautas y orientación operativa para la revisión ética de la investigación en salud con seres humanos.. [consultado 9 Junio 2018].

Disponible

en:

http://www.paho.org/hq/index.php?option=com docman\&task=doc view\&gid=22738\&lt emid=270\&lang=en.

Otzen T, Manterola C, Rodríguez I, García M (2011). La necesidad de aplicar el método científico en investigación clínica: problemas, beneficios y factibilidad del desarrollo de protocolos de investigación. International Journal of Morphology; 35(3), 1031-1036.

Oyarzún M, Pinto M E, Raineri G, Amigo H, Cifuentes L, González M J, et al. (2014) Experiencia del Comité de Ética de Investigación en Seres Humanos de la Facultad de Medicina de la Universidad de Chile y los desafíos que impone la nueva legislación chilena en la investigación médica. Rev Med Chile; 142, 889-95.

Oyarzún M., Raineri G (2013). Potencial impacto de la nueva legislación en la investigación biomédica en Chile. Rev Chil Enferm Respir; 29, 5-8.

Parab S, Bhalerao S (2010). Study designs. International Journal of Ayurveda Research;1(2), 128-131.

Pío XII. Discurso a los asistentes al I Congreso Internacional de Histopatología del Sistema Nervioso. Roma, 14 de septiembre de 1952.

Ramana K V, Kandi S, Boinpally PR (2013). Ethics in medical education, practice, and research: An insight. Ann Trop Med Public Health; 6, 599-602.

Reed W, Carroll J, Agramonte A, Lazear J (1900). The etiology of yellow fever-a preliminary note. Public Health Pap Rep; 26, 37-53.

Rodríguez Yunta, Eduardo (2004). Comités de evaluación ética y científica para la investigación en seres humanos y las pautas CIOMS 2002. Acta bioethica; 10(1), 3748.

Roy CC (1997). Survival of clinician scientist. Clinical and Investigative Medicine; 20: 283-9.

Ruiz J (2010). Importancia de la investigación. Rev. cient; 20(2), 125-125.

S Salas et al (2005). Médicos - Científicos en Chile: ¿una especie en extinción? Rev Méd Chile; 133, 121-128. 
Sanarelli G (1897). A lecture on yellow fever. With a description of the Bacillus Icteroides: Delivered before the University of Montevideo on June 10th, 1897. Br Med $\mathrm{J} ; 2,7-11$.

Savulescu J, Chalmers I, Blunt J (1996). Are research ethics committees behaving unethically? Some suggestions for improving performance and accountability. BMJ: British Medical Journal; 313(7069), 1390-1393.

Schroter S, Plowman R, Hutchings A, Gonzalez A (2006). Reporting ethics committee approval and patient consent by study design in five general medical journals. Journal of Medical Ethics; 32(12), 718-723.

Scott SD, Estabrooks CA, Allen M, Pollock C (2008). A context of uncertainty: How context shapes nurses' research utilization behaviors. Qualitative Health Research;18, 347-57.

Solimano G., Alarcón A., Sepúlveda N (2011). Estado del Arte de la investigación en Salud Pública en Chile 1989-2009. Informe de Resultados Programa Domeyko en Salud, Facultad de Medicina, Escuela de Salud Pública. Santiago de Chile.

Stevens B (2011). Health Clinician-Scientists in Academic Health Science Centres: Protecting an Endangered Species. Report on a National Symposium.

Tanne JH (2010). President Obama apologises to Guatemala over 1940 syphilis study. BMJ; 341, c5494.

Tejeda JG (2013). La universidad nerd. Revista chilena de literatura; (84): 155-160.

The Nuremberg Code (1947). BMJ 1996; 313, 1448.

UNESCO. Guía 2. Funcionamiento de los comités de bioética: procedimientos y políticas. 2006. [consultado 9 Junio 2018]. Disponible en: http://unesdoc.unesco.org/images/0014/001473/147392s.pdf.

Valenzuela S., Aliaga V., Burdiles P., et al. (2015). Reflexiones en torno a la ley $\mathrm{N}^{\circ}$ 20.584 y sus implicancias para la investigación biomédica en Chile. Rev Med Chile; 143, 96- 100.

Vargas J (2009). Ética, corrupción y burocracia. Perspectivas; 24, 209-226.

Vega J (2015). Efectos adversos graves de la ley $\mathrm{N}^{\circ}$ 20.584. Carta al editor. Rev Med Chile; 143, 682.

World Health Organization. Health research is essential for progress towards universal health coverage. [consultado 10 Junio 2018]. Disponible en: http://www.who.int/mediacentre/news/releases/2013/world health report 20130815/en l.

World Medical Association (1997). Declaration of Helsinki. JAMA; 277, 925-926.

X. Sierra (2011). Ética e investigación médica en humanos: perspectiva histórica. Actas Demorsifiliográficas;102 (6), 395-401. 